\title{
Perturbation amplitude in isocurvature inflation scenarios
}

\author{
Andrew R. Liddle and Anupam Mazumdar \\ Astrophysics Group, The Blackett Laboratory, Imperial College, London SW7 2BZ, United Kingdom
}

(Received 17 December 1999; published 12 May 2000)

\begin{abstract}
We make a detailed calculation of the amplitude of isocurvature perturbations arising from inflationary models in which the cold dark matter is represented by a scalar field which acquires perturbations during inflation. We use this to compute the normalization to large-angle microwave background anisotropies. Unlike the case of adiabatic perturbations, the normalization to COBE fixes the spectral index of the perturbations; if adiabatic perturbations are negligible then $n_{\text {iso }} \simeq 0.4$. Such blue spectra are also favored by other observational data. Although the pure isocurvature models are unlikely to adequately fit the entire observational data set, these results also have implications for models with mixed adiabatic and isocurvature perturbations.
\end{abstract}

PACS number(s): 98.80.Cq, 98.70.Vc

\section{INTRODUCTION}

There has been recent interest [1-6] in inflationary models which give rise to isocurvature perturbations in the Universe, rather than the traditional adiabatic ones. The simplest way to achieve this is to assume that the material presently making up the cold dark matter takes the form of a massive scalar field, which during inflation received perturbations by the usual mechanism. With suitable subsequent evolution, these perturbations can be the dominant ones in the present Universe.

The familiar inflationary calculation for adiabatic perturbations is extremely simple (see Ref. [7] for a review), because once the perturbations have been stretched to wavelengths greater than the Hubble length they become constant, and no calculation is required until the much later epoch when they cross back inside the Hubble radius, which for scales of interest is well after nucleosynthesis. By contrast, isocurvature perturbations $[8-10]$ are free to evolve on superhorizon scales, and the amplitude at the present day depends on the details of the entire cosmological evolution from the time that they are formed. On the other hand, because all super-Hubble radius perturbations evolve in the same way, the shape of the isocurvature perturbation spectrum is preserved during this evolution and so need not be calculated. In the literature, therefore, comparison with observation $[3,4]$ has largely focused on the spectral index of the perturbations, and it has been assumed that the model parameters could always be tweaked to ensure the amplitude is correct. However, as we will see the constraints on amplitude and slope are intimately related in these scenarios. In this paper, we compute the Sachs-Wolfe contribution to large-angle microwave background anisotropies for a particular class of isocurvature models which give non-Gaussian perturbations. Unlike the case of adiabatic perturbations, the Sachs-Wolfe effect fixes the slope of the isocurvature perturbations, as well as their amplitude, because the slope determines the matter density.

\section{MODEL DESCRIPTION}

The class of models we discuss is characterized by the cold dark matter being a scalar field with a time-dependent mass, which apart from its quantum fluctuations sits in the vacuum state during the entire history of the Universe. Particular implementations of that general statement have been given by Linde and Mukhanov [1] and by Peebles [2]. It is well known that a sufficiently massive scalar field $\phi$, with $m \gg H$ where $H$ is the Hubble parameter, oscillates with decaying amplitude such that its energy density $\rho_{\phi} \propto 1 / a^{3}$ where $a$ is the scale factor, and further that perturbations in this field obey the same equations as perturbations in a pressureless fluid. Such a scalar field is therefore a viable cold dark matter candidate, the classic example being the axion (see Ref. [10] for an early treatment and Ref. [11] for a review).

The mass of the scalar field in such a scenario must have quite a complicated evolution in order to generate a viable model.

\section{A. During inflation}

If the field is effectively massless, $m \ll H$, its perturbations will be scale-invariant. Such a spectrum is excluded by comparison of Cosmic Background Explorer (COBE) data with galaxy clustering; what is needed is a spectrum with a tilt favouring short-scale perturbations (usually called a blue spectrum). This can successfully be generated if $m \simeq H$ at the relevant stages of inflation [1]; the mass causes perturbations to die away outside the horizon, and those which have been outside the longest receive the greatest suppression. However if $m \gg H$ during inflation, then perturbations will be highly suppressed with an $n_{\text {iso }}=3$ spectrum, ${ }^{1}$ which is also unsatisfactory. One must therefore tune the mass to be close to $H$, perhaps best done by generating an effective mass $m_{\text {eff }}^{2}=\alpha H^{2}$ where $\alpha$ is some coupling hoped to be of order one.

\section{B. After inflation}

We are seeking to create isocurvature perturbations, of the type where the cold dark matter (CDM) perturbation is can-

\footnotetext{
${ }^{1} \mathrm{We}$ use conventions where $n_{\text {iso }}=0$ corresponds to scaleinvariance. Unfortunately the literature features at least three different conventions, where scale-invariance can correspond to an index of $-3,0$ or 1 .
} 
celled by the collective perturbation of all other species. In fact, the perturbations we are discussing are actually isothermal, since the fluctuations in $\phi$ are uncorrelated with those in the inflaton field which is what ultimately determines the radiation density after reheating. These isothermal perturbations can be broken into an adiabatic and an isocurvature part. However, as long as the energy density of the CDM field is totally subdominant to that of the inflaton at the end of inflation, the isocurvature perturbation will be by far the dominant part [2].

Another important point is that the inflaton must not decay into the CDM field, which would destroy the isocurvature perturbations, and so must decay only into conventional particles. Therefore at the end of inflation the CDM field has much less energy density than everything else, whereas by the present it has to dominate the baryonic component (e.g. the present Universe may have $\Omega_{\text {baryon }} \simeq 0.04, \Omega_{\phi} \simeq 0.3$ and a cosmological constant). While the field has a significant mass then $\rho_{\phi} \propto 1 / a^{3}$, as compared to the thermalized fluid which at early times goes as $\rho_{\text {rad }} \propto 1 / a^{4}$. However it turns out that a more dramatic gain is required, which is achieved by having an epoch where the mass is small enough to be negligible. When this happens, the scalar field feels no force and is effectively fixed, leading to a constant $\rho_{\phi}$ enabling it to "catch up" with the conventional matter.

\section{At late times}

In order to preserve the successes of the standard cosmology, the field preferably should already be acting as cold dark matter by the time of nucleosynthesis (though there may be no observational conflicts even if this behavior is delayed until sometime later). This requires that the mass is greater than the Hubble parameter at that time, and this guarantees that it will act this way when any astrophysically-interesting perturbations enter the horizon. Depending on whether one believes in a cosmological constant or not, the desired dark matter density is between about 5 and 20 times the baryon density, so the field must start to scale as $1 / a^{3}$ just when it reaches that relative density. This requires fine-tuning, but that's true of all dark matter models as they are all trying to explain the same coincidence of dark matter and baryon densities. The simplest scenario is that the mass is the bare mass of the field; the effective mass was large during inflation due to some coupling, and then the field evolved as effectively massless until $H$ fell to be of order this bare mass.

An interesting feature of this model is that the perturbations are crucial to define the background. In normal models there is a homogeneous background density $\rho_{\mathrm{CDM}}$ about which the perturbations are defined, but here the homogeneous background is $\phi=0 \Rightarrow \rho_{\phi}=0$. Instead, the mean density of the dark matter is generated by the perturbations, taking advantage of the density being proportional to the square of the field perturbation. At each point in space the field $\phi$ is oscillating with some amplitude. If we define $\Phi(\mathbf{x})$ to be the maximum of the oscillation at point $\mathbf{x}$, then the energy density is

$$
\rho_{\phi}(\mathbf{x})=\frac{1}{2} m^{2} \Phi^{2}(\mathbf{x})
$$

and the mean density is

$$
\bar{\rho}_{\phi}=\frac{1}{2} m^{2}\left\langle\Phi^{2}(\mathbf{x})\right\rangle=m^{2}\left\langle\phi^{2}(\mathbf{x})\right\rangle=m^{2} \int_{0}^{k_{\max }} \mathcal{P}_{\phi}(k) \frac{d k}{k} .
$$

In that last expression, $\mathcal{P}$ is the power spectrum of $\phi$, defined as

$$
\mathcal{P}_{\phi}(k)=\frac{k^{3}}{2 \pi^{2}}\left\langle\phi_{\mathbf{k}}^{*} \phi_{\mathbf{k}}\right\rangle,
$$

where $\phi_{\mathbf{k}}$ are the Fourier components of $\phi(\mathbf{x})$ and $k=|\mathbf{k}|$. We have included an upper cut-off $k_{\max }$, intended to be the shortest scale on which there are perturbations and estimated as the Hubble scale at the end of inflation.

The background dark matter density therefore has to be obtained self-consistently out of the perturbation calculation. Were the spectrum flat, this cannot be achieved; we need the power spectrum to rise sharply to short scales, as then the correct density can be obtained by suitable tuning of $k_{\max }$ and $m$ to give the observed value. Calculationally this is also desirable, as in considering the large-scale perturbations one can imagine smoothing out the small-scale perturbations to obtain a genuinely smooth background. It is interesting, though certainly not compelling, that the blue spectrum is favoured both in order to obtain the correct dark matter density and to match large-scale structure observations.

One should also note that the statistics of the perturbations are neither Gaussian nor strictly speaking chi-squared, though they are related to the latter. It is the total density $\rho_{\phi}$ which is chi-squared distributed, not the density contrast $\delta(\mathbf{x}) \equiv\left[\rho_{\phi}(\mathbf{x})-\bar{\rho}_{\phi}\right] / \bar{\rho}_{\phi}$.

\section{BACKGROUND AND PERTURBATION EVOLUTION}

The complete model needed to evaluate the amplitude of perturbations therefore has two inputs. The first is the evolution of the Hubble parameter $H(t)$. During inflation this is governed by the inflaton field, which is some other scalar field whose energy is dominating over the CDM field. After inflation, it is determined by whatever the matter content of the Universe is, and we shall be assuming the standard, simplest evolution of radiation domination after inflation giving way to matter domination. One could include a cosmological constant too but we will ignore that minor complication. The second input is the time-dependent mass of the CDM field. As discussed, this will be of order $H$ during the late stages of inflation, then at the end of inflation drops to a small constant value which is initially negligible. Then eventually the Hubble parameter drops below this value and the field begins truly to behave as CDM.

The perturbations need to be tracked at various stages. First the amplitude arising from quantum fluctuations needs to be computed. Subsequently, the perturbation is outside the Hubble radius, but still decays as it has a significant mass $m \sim H$. At the end of inflation the mass becomes negligible and the field freezes out, before eventually the mass becomes important and the field begins to evolve again. For all the 
earlier stages, it is simplest to define the evolution in terms of the scalar field itself. However, at the last stage we have exactly a standard isocurvature CDM model, and standard results (for example for the Sachs-Wolfe effect) can be applied. These are usually expressed not in terms of the field, but rather in terms of the entropy perturbation $S$ defined later on.

To begin with we need to study perturbations in the CDM field which is responsible for generating isocurvature fluctuations. The background evolution is governed by the inflaton field, which dictates that the Hubble expansion during inflation is $H^{2} \simeq V / 3 M_{\mathrm{Pl}}^{2}$, where $\mathrm{V}$ is the potential for the inflaton field and $M_{\mathrm{Pl}}=\sqrt{1 / 8 \pi G}$ is the reduced Planck mass. As explained earlier, we assume that energy density of the CDM field $\phi$ is subdominant compared to that of the inflaton during the slow-roll phase. We are interested in studying the fluctuations generated in the $\phi$ field. The fluctuations in the inflaton field cause perturbations in the curvature giving rise to adiabatic perturbations, but such perturbations are to be arranged to be negligible compared to the isocurvature ones.

There are various sources which would produce an effective mass to the CDM field [1]. One possibility is the existence of a time-varying coupling of the CDM to the inflaton field. During inflation such a coupling can be adjusted to give an effective mass to the CDM which is proportional to the Hubble parameter. Another possibility is the existence of a non-minimal coupling of the CDM to gravity. Such a term appears as a correction to the mass of the CDM and does not alter the dynamics of the overall evolution as the CDM field is oscillating at the bottom of the potential $\phi=0$. Such a coupling gives a correction to the mass of the CDM field: $m^{2}(H)=m^{2}+12 \xi H^{2}$ [1]. For our purposes, it does not matter what the source of the correction is as long as it fixes the effective mass to be of the order of the Hubble parameter. During inflation the presence of such a term provides the tilt in the power spectrum and just after the end of inflation it must switch off to avoid the CDM field dominating too early.

To study the perturbations we need the perturbation equation for the CDM

$$
\delta \ddot{\phi}{ }_{k}+3 H \delta \dot{\phi}_{k}+\left[\frac{k^{2}}{a^{2}}+m_{\mathrm{eff}}^{2}(t)\right] \delta \phi_{k}=0
$$

where the effective mass $m_{\text {eff }}(t)$ is

$$
m_{\mathrm{eff}}^{2}(t)=m^{2}+\alpha(t) H^{2},
$$

where $m$ is the bare mass term of the CDM field. We assume that $m^{2} \ll \alpha(t) H^{2}$ during inflation. The solution of Eq. (4) for $k \ll a H$ is well known [12]

$$
\delta \phi_{\mathbf{k}} \approx H(H a)^{-3 / 2}\left[\frac{k}{H a}\right]^{-\sqrt{9 / 4-m_{\mathrm{eff}}^{2} / H^{2}}} .
$$

If the ratio of effective mass $m_{\text {eff }}(t)$ and $H$ is constant during the entire period of inflation, and assuming $H$ is suitably slowly varying, then at the end of inflation the solution for the power spectrum for the modes which are already outside the horizon is [1]

$$
\mathcal{P}_{\delta \phi}(k) \equiv \frac{k^{3}}{2 \pi^{2}}\left|\delta \phi_{k}\right|^{2} \approx \frac{1}{2 \pi^{2}} H_{\mathrm{e}}^{2}\left(\frac{k}{H_{\mathrm{e}} a_{\mathrm{e}}}\right)^{2 \alpha / 3},
$$

where the subscript " $\mathrm{e}$ " denotes the end of inflation.

This final expression, Eq. (7), is strictly correct only at the end of inflation, and we have replaced $m_{\text {eff }}$ with $\alpha H^{2}$, where $\alpha$ is a constant less than one. An alternative physical scenario would be if $m_{\text {eff }}$ were constant rather than $\alpha$, in which case the tilt will pick up an extra scale-dependence, depending on the details of the explicit model. We will only consider the situation when $\alpha$ is a constant. Even in that case, an extra scale-dependence of the tilt arises because of variation of $H$ during inflation, but this contribution will be insignificant compared to the tilt induced by the mass.

After inflation ends, the effective mass must drop precipitously from its value during inflation to the bare mass $m$ which is then much less than $H$. Initially then the mass can be neglected, and Eq. (4) has a simple solution on large scales: $\delta \phi_{k}=$ const. As a result the power spectrum, Eq. (7), remains unchanged.

This simple situation does not hold for ever, as the Hubble parameter drops as $a^{-2}$ during radiation era, where $a$ is the scale factor of the Universe. Eventually the Hubble parameter becomes equal to the bare mass of $\phi$ and henceforth the mass of the $\phi$ field cannot be neglected in Eq. (4). From this time onwards, the CDM field acts like a true massive CDM field, and the amplitude of the perturbations falls as $a^{-3 / 2}$ during all subsequent phases of the Universe. Noting that $H(t)=H_{\mathrm{e}} a_{\mathrm{e}}^{2} / a^{2}$ during radiation domination, we find that at any time after mass turn on the spectrum of the fluctuations is given by

$$
\mathcal{P}_{\delta \phi}(k) \approx \frac{1}{2 \pi^{2}}\left(\frac{a}{a_{\mathrm{e}}}\right)^{-3}\left(\frac{H_{\mathrm{e}}}{m}\right)^{3 / 2} H_{\mathrm{e}}^{2}\left(\frac{k}{H_{\mathrm{e}} a_{\mathrm{e}}}\right)^{2 \alpha / 3}
$$

Preferably this transition should take place before the synthesis of light elements, when the ratio between the photon and the baryon is already a fixed constant, though compatibility with observations may still be maintained even if it happens some time afterwards. This expression for the spectrum continues to hold at all later epochs, including matter domination.

After mass turn-on, it is useful to consider an alternative quantity, the entropy perturbation $S$ given by

$$
S=\frac{\delta \rho_{\phi}}{\rho_{\phi}}-\frac{3}{4} \frac{\delta \rho_{\mathrm{r}}}{\rho_{\mathrm{r}}}
$$

where the subscript ' $r$ " denotes radiation. Because the perturbations are actually isothermal (albeit in a limit where 
isothermal and isocurvature are almost the same thing), we have $\delta \rho_{\mathrm{r}}=0$ and this can be written as ${ }^{2}$

$$
S=\frac{\delta \rho_{\phi}}{\rho_{\phi}} \equiv \frac{\rho_{\phi}(x)-\bar{\rho}_{\phi}}{\bar{\rho}_{\phi}}=\frac{\delta \phi^{2}(x)-\left\langle\delta \phi^{2}(x)\right\rangle}{\left\langle\delta \phi^{2}(x)\right\rangle},
$$

where $\bar{\rho}_{\phi}$ is the mean energy density and it is defined as the spatial average of the field fluctuations. $\bar{\rho}_{\phi}(x)$ defines the total energy density of the field $\phi$ at any given time and it is determined using Eq. (2) from the field fluctuations themselves. The advantage of using the entropy perturbation is that it is a standard result $[12,7]$ that on super-horizon scales it continues to be conserved even when the radiation is no longer dominant (at which point the radiation will acquire perturbations from the influence of the CDM field fluctuations on the expansion rate), and this conservation law can be used to evolve the perturbations up to decoupling to compute the Sachs-Wolfe effect.

Now we have all the necessary formulas to determine the Sachs-Wolfe effect for the isocurvature fluctuations of the $\phi$ field.

\section{COMPARISON WITH OBSERVATIONS}

\section{A. Normalization from the Sachs-Wolfe effect}

The anisotropy due to the entropy perturbation is given by the well-known formula $[9,13,7]$

$$
\frac{\Delta T(\mathbf{x})}{T}=-\frac{2 S}{5}
$$

Following the same steps as for the adiabatic case (see e.g. Ref. [7]), the spectrum of the $\mathrm{cmb}$, denoted by $C_{l}$, is given by

$$
C_{l}=\frac{16 \pi}{25} \int_{0}^{\infty} \frac{d k}{k} \mathcal{P}_{S}(k) j_{l}^{2}\left(\frac{2 k}{H_{0} a_{0}}\right),
$$

where $\mathcal{P}_{S}(k) \delta\left(\mathbf{k}-\mathbf{k}^{\prime}\right) \equiv\left\langle S_{\mathbf{k}}^{*} S_{\mathbf{k}^{\prime}}\right\rangle k^{3} / 2 \pi^{2}$, and $j_{l}$ is a spherical Bessel function. We have taken the distance to the lastscattering surface as $2 / a_{0} H_{0}$, where the subscript 0 corresponds to the present value. This formula is strictly true only for a critical-density universe, but could be readily generalized to low-density.

From Eq. (10), except for the $\mathbf{k}=0$ mode we have

$$
S_{\mathbf{k}}=\frac{1}{\left\langle\delta \phi^{2}\right\rangle}\left(\delta \phi^{2}\right)_{\mathbf{k}} \quad(\mathbf{k} \neq 0) .
$$

\footnotetext{
${ }^{2}$ The definition of $S$ in Eq. (9) is gauge-invariant. The approximation in Eq. (10) is not, but will hold in any reasonable gauge which has not been specifically chosen to make the scalar field perturbations unnaturally small (an example of such a pathological gauge would be to choose the spatial slices to make $\rho_{\phi}$ homogeneous). For instance, one can consider this expression to be in the longitudinal or comoving gauge. Having evaluated it in a gauge where the approximation is good, the result holds in all gauges.
}

Hence

$$
\mathcal{P}_{S}(k)=\frac{1}{\left\langle\delta \phi^{2}\right\rangle^{2}} \mathcal{P}_{\delta \phi^{2}}(k)=\frac{m^{4}}{\bar{\rho}_{\phi}^{2}} \mathcal{P}_{\delta \phi^{2}}(k) .
$$

To evaluate $C_{l}$ we need to compute the power spectrum of $\delta \phi^{2}$. Its calculation is quite subtle, and we carry it out in the Appendix. The result, for the range of tilts we are interested in, is

$$
\mathcal{P}_{\delta \phi^{2}}=\frac{I(2 \alpha / 3)}{4 \pi^{4}}\left(\frac{a_{e}}{a}\right)^{6}\left(\frac{H_{e}}{m}\right)^{3} \frac{H_{e}^{4}}{1-2 \alpha / 3}\left(\frac{k}{H_{e} a_{e}}\right)^{4 \alpha / 3}
$$

where $I(2 \alpha / 3)$ is a constant described in the Appendix, which is of order 10 for the values of $\alpha$ we will be interested in. Here $H_{\mathrm{e}}$ is the Hubble parameter at the end of inflation.

Substituting Eq. (15) into Eq. (12), we get the following expression:

$$
\begin{aligned}
C_{l}= & \frac{4 I(2 \alpha / 3) m^{4}}{25 \pi^{3} \bar{\rho}_{\phi}^{2}}\left(\frac{a}{a_{\mathrm{e}}}\right)^{-6}\left(\frac{H_{\mathrm{e}}}{m}\right)^{3} \frac{H_{\mathrm{e}}^{4}}{1-2 \alpha / 3} \\
& \times \frac{1}{\left(H_{\mathrm{e}} a_{\mathrm{e}}\right)^{4 \alpha / 3}} \int_{0}^{\infty} d k k^{4 \alpha / 3} j_{l}^{2}\left(\frac{2 k}{H_{0} a_{0}}\right) .
\end{aligned}
$$

Equation (16) can be further simplified by performing the integration

$$
\begin{aligned}
l(l+1) C_{l}= & \frac{2 I(2 \alpha / 3) m^{4} H_{\mathrm{e}}^{4}}{25 \pi^{3} \bar{\rho}_{\phi}^{2}(1-2 \alpha / 3)} \\
& \times \frac{a_{\mathrm{e}}^{6}}{a^{6}} \frac{H_{\mathrm{e}}^{3}}{m^{3}}\left(\frac{H_{0} a_{0}}{2 H_{\mathrm{e}} a_{\mathrm{e}}}\right)^{4 \alpha / 3}\left\{\frac{\sqrt{\pi}}{2} l(l+1)\right. \\
& \left.\times \frac{\Gamma[(2-4 \alpha / 3) / 2] \Gamma[l+2 \alpha / 3]}{\Gamma[(3-4 \alpha / 3) / 2] \Gamma[l+(2-2 \alpha / 3)]}\right\} .
\end{aligned}
$$

In fact, as long as $l \gg 1$ and $l \gg 2 \alpha / 3$, the complicated term within the braces can be approximated simply by $l^{4 \alpha / 3}$ (see e.g. Ref. [7]).

Although in this expression the quantities $\bar{\rho}_{\phi}$ and $a$ were given at the time of decoupling, since $\bar{\rho}_{\phi} \propto 1 / a^{3}$ they can in fact be evaluated at any epoch, including the present.

Next we can estimate $\bar{\rho}_{\phi}$ by using Eqs. (2) and (8), obtaining

$$
\bar{\rho}_{\phi}=\frac{3 m^{2}}{4 \pi^{2} \alpha}\left(\frac{a}{a_{\mathrm{e}}}\right)^{-3}\left(\frac{H_{\mathrm{e}}}{m}\right)^{3 / 2} H_{\mathrm{e}}^{2}\left(\frac{k_{\mathrm{max}}}{H_{\mathrm{e}} a_{\mathrm{e}}}\right)^{2 \alpha / 3}
$$

Substituting $\bar{\rho}_{\phi}$ in Eq. (17), and taking $k_{\max } \sim H_{\mathrm{e}} a_{\mathrm{e}}$ since we assume that the shortest scale perturbations generated are at the horizon scale at the end of inflation, one gets a simple relation for a multipole of order $l$, namely 


$$
l(l+1) C_{l} \approx \frac{32 \pi}{255} \frac{I(2 \alpha / 3) \alpha^{2}}{1-2 \alpha / 3}\left[\frac{l H_{0} a_{0}}{2 H_{\mathrm{e}} a_{\mathrm{e}}}\right]^{4 \alpha / 3} .
$$

The COBE observations [14] give the 10th multipole as $l(l+1) C_{l} \sim 7 \times 10^{-10}$. Taking the ratio of Hubble scales at the present and at the end of inflation to be given by the usual estimate $\ln \left(H_{\mathrm{e}} a_{\mathrm{e}} / H_{0} a_{0}\right) \approx 50$, this gives

$$
n_{\mathrm{iso}} \simeq \frac{4 \alpha}{3} \simeq 0.43
$$

where $n_{\text {iso }}$ refers to the slope of $\mathcal{P}_{S}$.

Predominantly, then, the Sachs-Wolfe effect for isocurvature perturbations fixes the slope of the perturbations, rather than the amplitude as one finds for adiabatic perturbations. The reason is that the slope, favouring short wavelengths, is what determines the matter density, and for the observed perturbations $S \simeq 10^{-5}$ to be achieved, the tilt must be steep enough to favor short-scale perturbations in determining the matter density against which the perturbations are observed. Were the spectrum flatter, then the background density would not be high enough, whereas if it were steeper than the large-scale perturbations would not give enough density contrast against the mean background derived from the short scales. This argument was given in Ref. [1]; our calculation had given a precise quantification of the constraint.

It is intriguing that the slope required to give the correct magnitude of the Sachs-Wolfe effect is also in better agreement as to the slope of the large-angle $C_{l}$ than a flat spectrum, although it seems unlikely that a pure isocurvature model will be able to fit the complete set of observational data $[15,4]$.

\section{B. The CDM matter density}

We can constrain the bare mass $m$ of the CDM from the present density

$$
\bar{\rho}_{\phi} \simeq \rho_{\text {crit }}=3 H_{0}^{2} M_{\mathrm{Pl}}^{2},
$$

where we continue to assume that we live in a criticaldensity universe. With the help of Eq. (18) we can derive a simple expression for the CDM mass

$$
m=\left(4 \pi^{2} \alpha\right)^{2} M_{\mathrm{Pl}}^{4} \frac{H_{0}^{4}}{H_{\mathrm{e}}^{7}}\left(\frac{a_{0}}{a_{\mathrm{e}}}\right)^{6},
$$

where we took $k_{\max }=H_{\mathrm{e}} a_{\mathrm{e}}$. To simplify this, we model the Universe as matter-dominated during reheating until $H_{\mathrm{rh}}$, followed by the usual cosmology. During matter domination $a \propto H^{-2 / 3}$ and during radiation domination $a \propto H^{-1 / 2}$, leading to

$$
m=16 \pi^{4} \alpha^{2} M_{\mathrm{Pl}}^{4} \frac{H_{0}^{4}}{H_{\mathrm{e}}^{7}}\left(\frac{a_{0}}{a_{\mathrm{eq}}} \frac{a_{\mathrm{eq}}}{a_{\mathrm{rh}}} \frac{a_{\mathrm{rh}}}{a_{\mathrm{e}}}\right)^{6}=16 \pi^{4} \alpha^{2} M_{\mathrm{Pl}}^{4} \frac{H_{\mathrm{eq}}}{H_{\mathrm{rh}} H_{\mathrm{e}}^{3}} .
$$

For critical density the Hubble parameter at matter-radiation equality is $H_{\mathrm{eq}}=5.25 \times 10^{6} h^{3} H_{0}$ where $H_{0}=2.1 \mathrm{~h} \times 10^{-42}$ $\mathrm{GeV}$ (with $0.5<h<0.75$ ) is the present Hubble parameter. Putting in characteristic values leads to

$$
\frac{m}{1 \mathrm{GeV}}=44 \alpha^{2} g_{*}^{-1 / 2} h^{4}\left(\frac{10^{13} \mathrm{GeV}}{H_{\mathrm{e}}}\right)^{3}\left(\frac{10^{10} \mathrm{GeV}}{T_{\mathrm{rh}}}\right)^{2},
$$

where $g_{*} \sim 100$ is the number of particle species at reheating.

The model appears compatible with observations for a reasonable range of parameters. First, gravitational wave production requires $H_{e}<10^{14} \mathrm{GeV}$, or gravitational waves would give the dominant contribution to the COBE anisotropies (see e.g. [7]). Indeed, it will need to be some way less than this to make sure that adiabatic perturbations do not exceed the isocurvature ones which we presume to dominate. The mass $m$ needs to be less than $H_{\mathrm{e}}$ for the scenario to be consistent, and should not turn on too late if the standard cosmology is to be recovered. Everything would be completely safe if turn on precedes nucleosynthesis, but may continue to be permitted even if turn on is later, since the dark matter density would be negligible during nucleosynthesis. However, since $H_{\text {nucl }} \simeq 10^{-24} \mathrm{GeV}$ this is unlikely to be the situation. Finally, if ones assumes supersymmetry then the reheat temperature is constrained by gravitino production from two-body scattering processes in the thermal bath. If the reheat temperature is too high then gravitinos are produced with an abundance which destroys standard nucleosynthesis. To avoid this, it should satisfy be $T_{\mathrm{rh}} \leqslant 10^{10}$ $\mathrm{GeV}$ [16]. This implies reheating should be inefficient, though the problem is no different here to usual inflation models, and may indeed be less severe than some standard models as we can accommodate a low energy density at the end of inflation.

Parameters exist which satisfy all these constraints. For example, $H_{\mathrm{e}} \simeq 10^{12} \mathrm{GeV}, T_{\mathrm{rh}} \simeq 10^{9} \mathrm{GeV}$ and $m \sim 10^{4} \mathrm{GeV}$ is a suitable combination.

\section{DISCUSSION AND CONCLUSIONS}

We have carried out a full calculation of the amplitude of microwave anisotropies within a class of isocurvature inflation models which includes models by Linde and Mukhanov [1] and by Peebles [2]. We have not dwelt on the modelbuilding issues of obtaining the appropriate time dependence of the CDM field's mass, but under the assumption that it can be made to behave as required we have evaluated the model constraints. The key result is that the Sachs-Wolfe effect in these models primarily constrains the slope, rather than the amplitude, of the perturbations. Typically observational comparisons have assumed that the slope is a free parameter (e.g. [4]), without taking this additional restriction into account.

A broad range of parameters appears capable of simultaneously matching the COBE observations and the required CDM matter density. It is intriguing that the slope $n_{\text {iso }} \simeq 0.4$ 
required to match the Sachs-Wolfe amplitude is also in the right vicinity to have a chance of matching the slope of the large-angle anisotropy spectrum. Nevertheless, it is probably true that such pure isocurvature scenarios are unable to fit the complete data set $[15,4]$. There does however remain scope for mixed adiabatic and isocurvature scenarios $[15,6,17,4]$, and our results can be applied, with minor modification, in those scenarios too.

\section{ACKNOWLEDGMENTS}

A.M. is supported by Inlaks foundation. We thank Antonio da Silva, Lev Kofman, David Lyth and David Wands for useful discussions.

\section{APPENDIX: THE POWER SPECTRUM OF $\delta \phi^{2}$}

The entropy perturbation is given by the square of the field perturbation, and so we need the spectrum of that quantity. We assume that the power spectrum of $\delta \phi$ itself is given by

$$
\mathcal{P}_{\delta \phi}(k)=A\left(\frac{k}{H_{\mathrm{e}} a_{\mathrm{e}}}\right)^{n}
$$

The spectrum of $\delta \phi^{2}$ is given by $[18]^{3}$

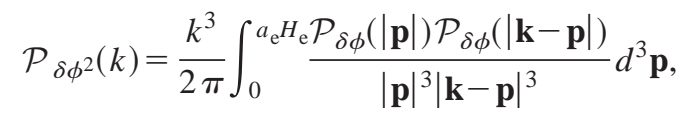

where $|\mathbf{p}|$ and $|\mathbf{k}-\mathbf{p}|$ are the wave numbers. This integral needs to be carried out with some care concerning the angular part. We can use rotational symmetry to align $\mathbf{k}$ along the $z$-axis to make $|\mathbf{k}-\mathbf{p}|$ independent of the azimuthal angle, so that $d^{3} \mathbf{p} \equiv 2 \pi p^{2} \sin \theta d \theta d p$. Substituting in the spectrum from Eq. (A1) gives

$$
\mathcal{P}_{\delta \phi^{2}}=\frac{k^{3} A^{2}}{\left(a_{\mathrm{e}} H_{\mathrm{e}}\right)^{2 n}} \int_{0}^{a_{\mathrm{e}} H_{\mathrm{e}}} \int_{0}^{\pi} p^{n-1}|\mathbf{k}-\mathbf{p}|^{n-3} \sin \theta d \theta d p,
$$

where $p \equiv|\mathbf{p}|$ and $k=|\mathbf{k}|$. Using

$$
|\mathbf{k}-\mathbf{p}|^{2}=|\mathbf{k}|^{2}+|\mathbf{p}|^{2}-2|\mathbf{k}||\mathbf{p}| \cos \theta,
$$

leads to the integral

\footnotetext{
${ }^{3}$ In Ref. [18] an unusual mix of Fourier conventions is used, but
} the final result is correct.

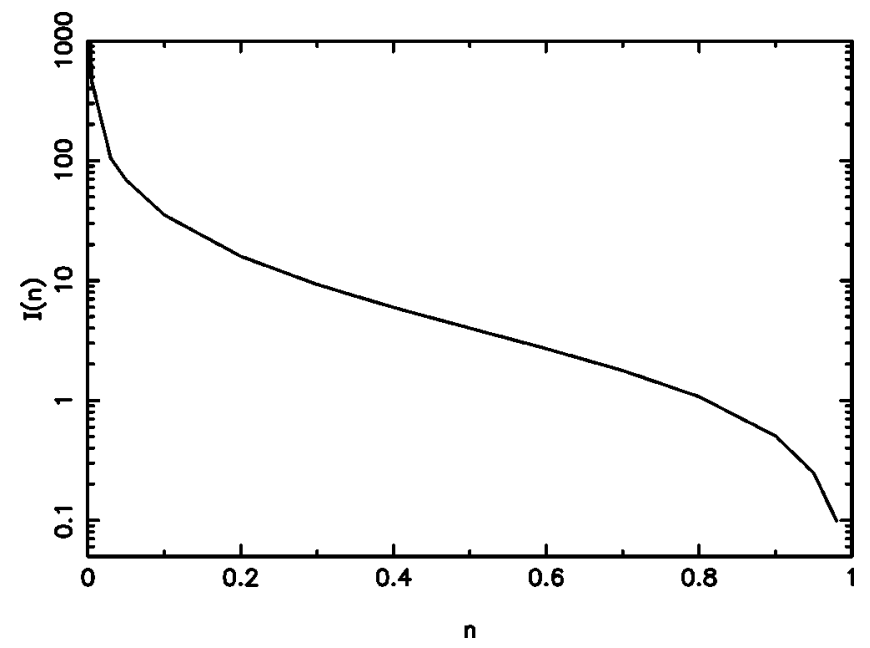

FIG. 1. The result of numerical evaluation of the integral of Eq. (A8), for $n$ in the range zero to one.

$$
\begin{aligned}
\mathcal{P}_{\delta \phi^{2}}(k)= & \frac{k^{2} A^{2}}{\left(a_{\mathrm{e}} H_{\mathrm{e}}\right)^{2 n}} \frac{1}{1-n} \int_{0}^{a_{\mathrm{e}} H_{\mathrm{e}}} p^{n-2} \\
& \times\left[|k-p|^{n-1}-(k+p)^{n-1}\right] d p .
\end{aligned}
$$

Finally, setting $u=p / k$ gives

$$
\begin{aligned}
\mathcal{P}_{\delta \phi^{2}}(k)= & \frac{A^{2}}{1-n}\left(\frac{k}{a_{\mathrm{e}} H_{\mathrm{e}}}\right)^{2 n} \int_{0}^{a_{\mathrm{e}} H_{\mathrm{e}} / k} u^{n-2} \\
& \times\left[|1-u|^{n-1}-(1+u)^{n-1}\right] d u,
\end{aligned}
$$

where we are interested in $n$ positive, and usually less than one. This final integral has interesting properties. It is convergent as $u \sim 0$. For $n<1$ the integrand is divergent at $u$ $=1$ (i.e. $p=k$ ) but it is integrable. Finally, at large $u$ the integrand goes as $u^{2 n-4}$. If $n \geqslant 3 / 2$, the upper limit dominates and one finds $\mathcal{P}_{\delta \phi^{2}} \propto k^{3}$; this limit appears for instance in the analysis of preheating models in Ref. [19] where $n=3$. However for the smaller $n$ values we are interested in, the integral is dominated by $u \sim 1$. The upper limit of the integral becomes irrelevant and can be taken to infinity for modes with $k \leqq a_{\mathrm{e}} H_{\mathrm{e}}$, so that the integral is independent of $k$. The spectrum therefore has tilt $\mathcal{P} \delta \phi^{2}(k) \propto k^{2 n}$. For the range of $n$ we will be interested in, $0<2 n<1$, we can write

$$
\mathcal{P}_{\delta \phi^{2}}(k)=\frac{A^{2} I(n)}{1-n}\left(\frac{k}{a_{\mathrm{e}} H_{\mathrm{e}}}\right)^{2 n},
$$

where

$$
I(n)=\int_{0}^{\infty} u^{n-2}\left[|1-u|^{n-1}-(1+u)^{n-1}\right] d u .
$$

We are not aware of an analytical evaluation of this integral except for special cases, but it is readily done numerically and we show the result in Fig. 1. 
[1] A. D. Linde and V. Mukhanov, Phys. Rev. D 56, 535 (1997).

[2] P. J. E. Peebles, Astrophys. J. 510, 523 (1999).

[3] P. J. E. Peebles, Astrophys. J. 510, 531 (1999).

[4] E. Pierpaoli, J. García-Bellido, and S. Borgani, J. High Energy Phys. 10, 015 (1999).

[5] S. Mollerach and S. Matarrese, Phys. Rev. D 45, 1961 (1992); M. Bucher, K. Moodley, and N. Turok, astro-ph/9904231; W. Hu and P. J. E. Peebles, Astrophys. J. Lett. 528, L61 (2000).

[6] D. Langlois, Phys. Rev. D 59, 123512 (1999).

[7] A. R. Liddle and D. H. Lyth, Phys. Rep. 231, 1 (1993).

[8] A. D. Linde, Phys. Lett. 158B, 375 (1985); A. D. Linde and L. A. Kofman, Nucl. Phys. B282, 555 (1987); H. Kodama and M. Sasaki, Int. J. Mod. Phys. A 2, 491 (1987); W. Hu and N. Sugiyama, Phys. Rev. D 51, 2599 (1995).

[9] G. Efstathiou and J. R. Bond, Mon. Not. R. Astron. Soc. 218, 103 (1986).

[10] L. Kofman, Phys. Lett. B 173, 400 (1986).
[11] E. W. Kolb and M. S. Turner, The Early Universe (AddisonWesley, Redwood City, CA, 1990).

[12] V. F. Mukhanov, H. A. Feldman, and R. H. Brandenberger, Phys. Rep. 215, 203 (1992).

[13] A. A. Starobinsky, Pis'ma Astron. Zh. 14, 394 (1988) [Sov. Astron. Lett. 14, 166 (1988)].

[14] C. L. Bennett et al., Astrophys. J. Lett. 464, L1 (1996).

[15] R. Stompor, K. M. Górski, and A. J. Banday, Astrophys. J. 463, 8 (1996).

[16] S. Sarkar, Rep. Prog. Phys. 59, 1493 (1996).

[17] M. Kawasaki, N. Sugiyama, and T. Yanagida, Phys. Rev. D 54, 2442 (1996); K. Enqvist and H. Kurki-Suonio, ibid. 61, 043002 (2000).

[18] D. H. Lyth, Phys. Rev. D 45, 3394 (1992).

[19] A. R. Liddle, D. H. Lyth, K. Malik, and D. Wands, Phys. Rev. D 61, 103509 (2000). 